\title{
DONNÉES PRÉLIMINAIRES \\ SUR LA FAUNE PLANCTONIQUE ET BENTHIQUE DE DEUX ETANGS PISCICOLES DE LA DOMBES (AIN)
}

par C. Amoros et J. JugeT.

Une étude écologique des étangs piscicoles de la Dombes est actuellement en cours. Au programme de recherches figure l'étude de l'évolution des populations planctoniques et benthiques de trois étangs de la Réserve Biologique de Villars-les-Dombes (Ain).

Un inventaire systématique préliminaire de quelques groupes d'Invertébrés particulièrement représentatifs de la faune planctonique et benthique, les Cladocères, les Copépodes, les Nématodes et les Oligochètes, fait l'objet de la présente note. Cet inventaire est circonscrit à deux étangs de la Réserve : l'étang Petit-Turlet et l'étang Bogue (voir figure 1 et planche 1), prospectés régulièrement au cours de l'année 1969 et dont les caractères seront résumés dans un prochain paragraphe.

Aucun des travaux antérieurs relatifs aux étangs de la Dombes n'a trait aux Helminthes; par contre, en ce qui concerne les Entomostracés, des listes faunistiques ont été publiées par Pelosse [1926], Gauthier [1931] ainsi que par Vivier el Wurtz [1960].

\section{1. - IL MILIEU}

Sous un climat de tendance continentale, avec des précipitations de l'ordre de 750 à $1100 \mathrm{~mm}$ par an, le plateau dombiste s'étend au Nord-Est de Lyon, à une altitude moyenne de $290 \mathrm{~m}$. Au centre de ce plateau, sur un lehm imperméable et siliceux, ont été créés les étangs de pisciculture.

Ces étangs sont vidés tous les ans pour la pêche et cette vidange est traditionnellement suivie une année sur trois d'un assèchement et d'une mise en culture du sol. Dans chaque étang est creusé un chenal central dont la profondeur peut atteindre 2 mètres alors (que la profondeur moyenne des étangs est de l'ordre de 1 mètre. Outre leur mode d'exploitation original [Wurtz, 1956 et 1960, MAs, 1959, Levrat, 1964-1965| les étangs dombistes se caractérisent 
par l'acidité et la pauvreté de leurs eaux en sels minéraux dissous et en particulier en calcium [WuRTz, 1962].

Les deux étangs prospectés en 1969 et dont quelques caractères physiques et chimiques sont résumés dans le Tableau I, bien qu'assez analogues par certains caractères, présentent cependant quelques différences. Leur profondeur est du même ordre de grandeur (de l'ordre de $2 \mathrm{~m}$ au niveau de la vanne de vidange) mais Petit-Turlet s'étend sur près de 30 ha alors que les eaux de Bogue ne couvrent que 5 à 6 ha $^{1}$. D'autre part, Petit-Turlet a été asséché et cullivé en avoine en 1966 tandis que le dernier assec de Bogue remonte à une quinzaine d'années.

TABdead 1. - Quelques caractères physiques et chimiques de l'eau.

\begin{tabular}{|c|c|c|c|c|}
\hline \multirow{2}{*}{ Facteurs } & \multicolumn{2}{|c|}{$\begin{array}{c}\text { Etang Petit- } \\
\text { Turlet }\end{array}$} & \multicolumn{2}{|c|}{$\begin{array}{l}\text { Etang } \\
\text { Bogue }\end{array}$} \\
\hline & $\min$. & $\max$. & $\min$. & $\max$. \\
\hline Température $\left({ }^{\circ} \mathrm{C}\right) \dot{\mathrm{a}}-0,5 \mathrm{~m}$ & 1 & 31 & 1 & 31 \\
\hline Transparence (m) & 0.1 & 2.0 & 0.15 & 1.5 \\
\hline $\mathrm{pH}$ & 6.6 & 7.3 & 6.0 & 6.7 \\
\hline Calcium (mg/l de $\mathrm{Ca}++)$ à $-0,5 \mathrm{~m}$ & 10.0 & 15.0 & 3.5 & 6.5 \\
\hline Oxygène lissous $(\mathrm{mg} / \mathrm{l})$ à $-0,5 \mathrm{~m}$ & 4.0 & 12.0 & 0.5 & 10.8 \\
\hline
\end{tabular}

Les eaux de Bogue sont toujours moins oxygénées, plus pauvres en calcium dissous et plus acides. Cette acidité et l'absence d'assec expliqueraient l'abondance de matières humiques non ou très partiellement dégradées sur le fond de Bogue et la plus faible teneur en matières organiques de ce sédiment (BEaupied, 1969, dose par la méthode Anne 1,7\% de matières organiques dans le sédiment de Bogue le 12-V-66 et 2,4 \% dans Petit-Turlet le 6-IX-65).

L'évolution naturelle de cet étang se traduit aussi par la prolifération des Macrophytes alors que dans Petit-Turlet leur développement tend à être restreint aux zones de faible profondeur. La végétation littorale représentée surtout par Scirpus lacustris accompagné de Glyceria fluitans, Phalaris arundinacea, Sparganium simplex, Iris pseudacorus et Typha latifolia, occupe une très grande partic de Bogue, cependant que la végétation flottante (Trapa natans et Potamogeton natans essentiellement) envahit en été toute la nappe d'eau (BEAUPIED, comm. pers.).

1. Les surfaces cadastrales représentent respectivement 31 ha et 10 ha pour les deux étangs. 


\section{Planche I}

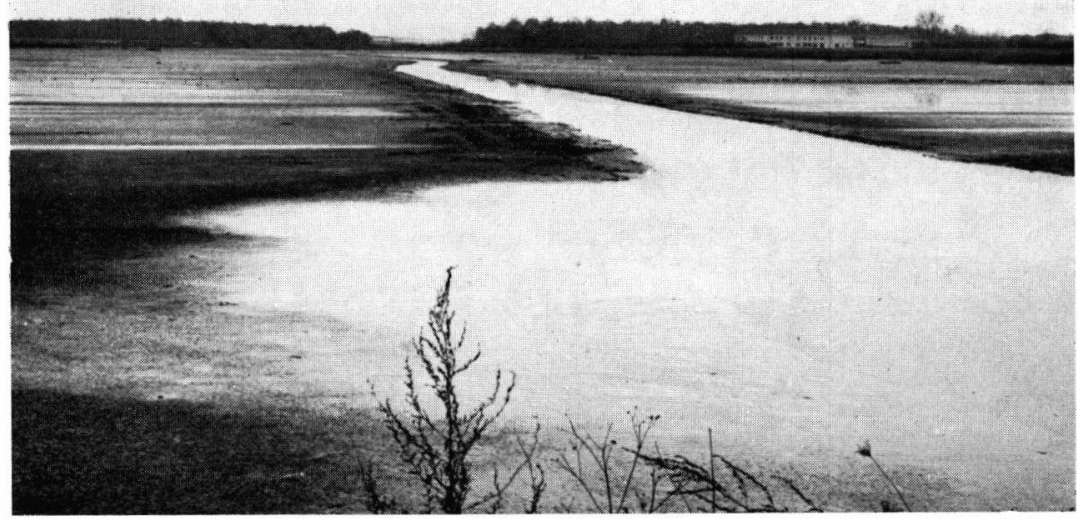

1. - L'étang Petit-Turlet vu de la route N.83, en période de vidange pour la récolte du poisson (novembre 1969); au centre le bief de drainage; à l'arrière-plan à droite, les bâtiments de Grange-Neuve, abritant les Services Administratifs et les Laboratoires.

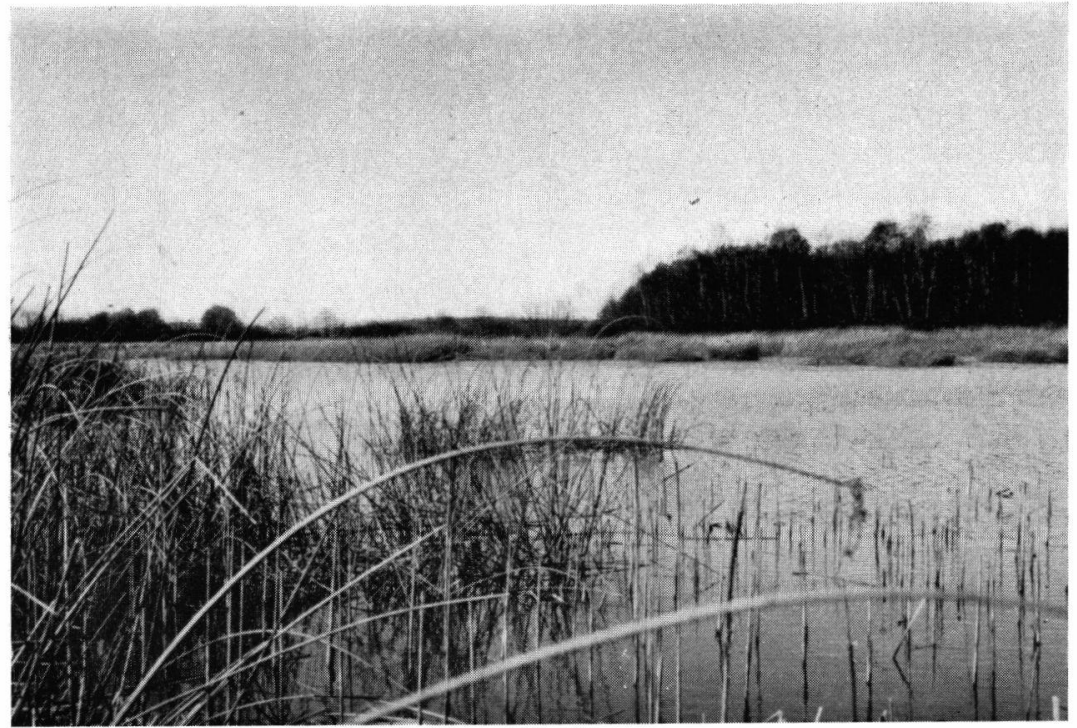

2. - L'étang Bogue en hiver; à l'arrière-plan, à droite, le bois Grand-Taillis. 
En ce qui concerne la pisciculture, alors que dans Petit-Turlet engraissent Tanches, Blancs (Gardons et Rotengles), Brochets et surtout Carpes, Bogue, considéré comme moins productif, sert à l'alevinage.

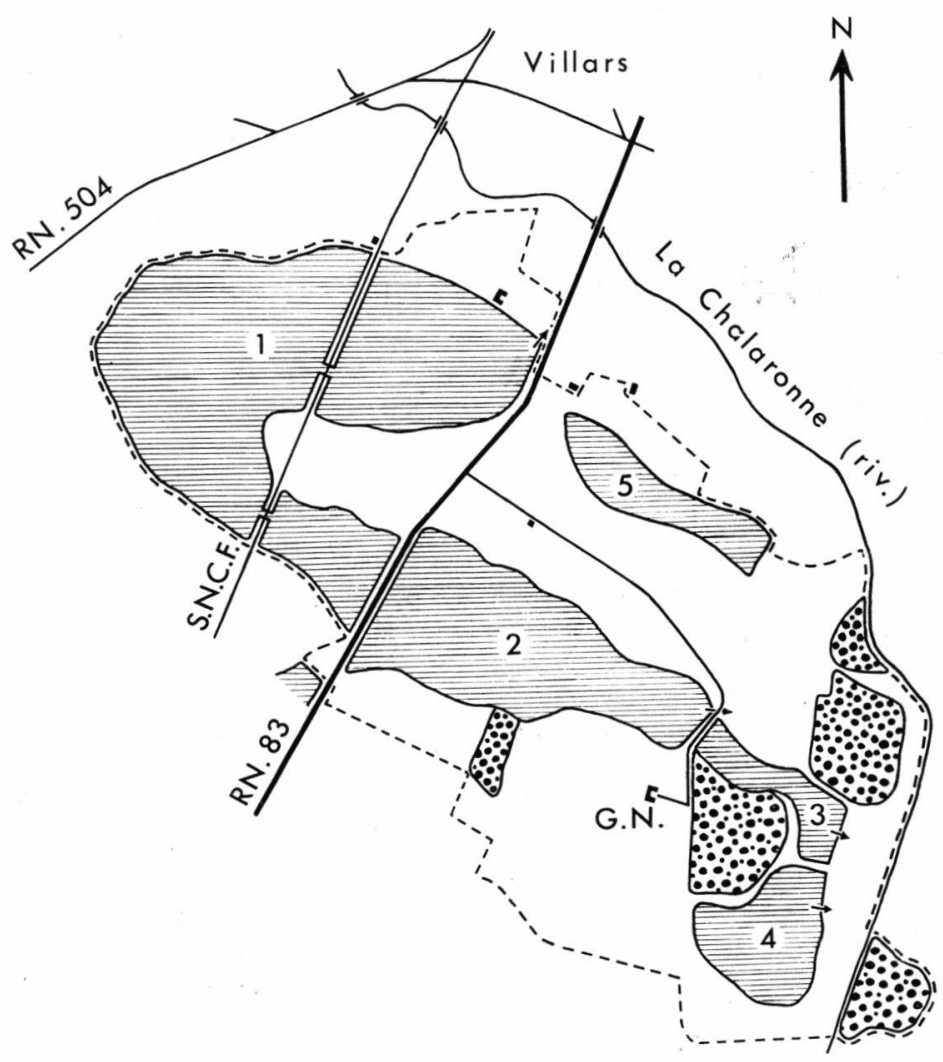

Etangs

a:: Bois

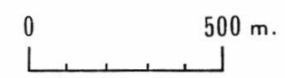

Fig. 1. - Les étangs de la Réserve Biologique de Dombes. 1 : étang GrandTurlet; 2 : étang Petit-Turlet; 3 : étang Sainte-Anne; 4 : étang Bogue; 5 : étang Bel-Air (Parc Zoologique); G.N. : ferme de Grange-Neuve; RN 83 : route nationale $\mathrm{n}^{\circ} 83$; S.N.C.F. : voie ferrée.

\section{2. - LA FAUNE}

\section{- Techniques de prélèvement.}

Le zooplancton est recueilli à l'aide d'un filet bicônique en gaze à bluter dont le vide de maille moyen est de 240 microns. Ce filet est traîné par une embarcation, le plus souvent dans le chenal 
central. Nous utilisons en outre une bouteille de Van Dorn de 7 litres pour récolter le matériel destiné aux analyses quantitatives.

La macrofaune benthique est prélevée au moyen d'une benne d'Fkman (de $15 \times 15 \mathrm{~cm}$ d'ouverture); le microbenthon est récupéré par carottage. L'extraction de la faune du sédiment s'obtient par tamisage sous l'eau à l'aide d'un tamis inox dont le vide de maille est de 250 microns pour le macrobenthon et de 40 microns pour le microbenthon.

\section{Les Cladocères ${ }^{1}$}

TABLEAU 2

\begin{tabular}{|c|c|c|}
\hline & Petit-Turlet & Bogue \\
\hline \multicolumn{3}{|l|}{ — Famille Sididae : } \\
\hline $\begin{array}{l}\text { Sida crystallina (O. F. Müller) } \\
\text { Diaphanosoma brachyurum Liéven }\end{array}$ & $\begin{array}{l}+ \\
+\end{array}$ & + \\
\hline \multicolumn{3}{|l|}{ - Famille Daphniidae : } \\
\hline $\begin{array}{l}\text { Daphnia longispina O.F. Müller } \\
\text { Daphniat ambigua Scourfield }\end{array}$ & + & + \\
\hline Simocephalus vetulus (O. F. Müller) & + & + \\
\hline Ceriodaphnia reticulata (Jurine) & + & + \\
\hline Ceriodaphnia pulchella Sars & + & + \\
\hline $\begin{array}{l}\text { - Famille Moinidae : } \\
\text { Moina micrura Kurz }\end{array}$ & —Famille Moinidae: & \\
\hline \multicolumn{3}{|l|}{$\begin{aligned} \text { - Famille Bosminidae : } & \text { Bosmina longirostris (O. F. Müller) }\end{aligned}$} \\
\hline $\begin{array}{l}\text { - Famille Macrothricidae : } \\
\text { Ilyocryptus agilis Kur\% }\end{array}$ & — Famille Macrothricidae : & \\
\hline \multicolumn{3}{|l|}{ - Famille Chydoridae : } \\
\hline $\begin{array}{l}\text { Eurycercus lamellatus (O. F. Müller) } \\
\text { Acroperus harpae Bairdl }\end{array}$ & $\begin{array}{l}+ \\
+\end{array}$ & . \\
\hline $\begin{array}{l}\text { Acroperus harpae Baird } \\
\text { Leydigia leydigi Schödler }\end{array}$ & $\begin{array}{l}+ \\
+\end{array}$ & $\begin{array}{l}+ \\
+\end{array}$ \\
\hline Alona rectangula Sars & + & + \\
\hline Alona affinis Leydig & + & + \\
\hline Alona guttata Sars & & + \\
\hline Alonella nana (Baird) & + & + \\
\hline Rhynchotalona rostrata (Koch) & + & + \\
\hline Pleuroxus trigonellus (O. F. Müller) & + & \\
\hline Chydorus sphaericus (O. F. Müller) & + & + \\
\hline
\end{tabular}

1. Nous nous référons à Gous.dex (1968) pour la détermination de Moina micrura et à Scounfield et Harding (1958) pour la détermination des autres Cladocères.

Nous tenons à remercier $M^{m e} J$. REy qui a bien voulu vérifier nos déterminations de Cladocères. 
- Sida crystallina, Simocephalus vetulus et Ceriodaphnia reticulata qui vivent habituellement parmi les végétaux, sont assez rares dans les prises de plancton.

- Laphnia longispina et Bosmina longirostris sont deux espèces planctoniques communes que l'on rencontre pratiquement toute l'année dans les deux étangs.

- On ne trouve l'espèce Diaphanosoma brachyurum qu'en été; elle est alors abondante dans les eaux de Petit-Turlet.

- Daphnia ambigua que nous avons recueillie dans le plancton de Petit-Turlet, de janvier à mars 1969, n'avait pas encore été signalée en France.

- Ceriodaphnia pulchella est très bien représentée dans les eaux de Petit-Turlet en été et en automne. Quoique moins abondante, elle est aussi présente à Bogue.

- Moina micrura signalée dans un petit lac du Sud-Ouest de la France par REY [1966] serait une forme sténotherme d'eau chaude. Nous l'avons rencontrée en abondance dans les eaux de Petit-Turlet à la fin de l'été.

- Ilyocryptus agilis, espèce typiquement benthique, a été capturé sur le fond de Petit-Turlet.

- La plupart des Chydoridae vivent également sur le fond ou bien parmi les Phanérogames, ils sont en général très peu représentés dans nos prélèvements de plancton.

\section{Les Copépodes ${ }^{1}$}

- Diaptomus castor n'est présent qu'au début du printemps seulement.

- Aucun Calanoida n'est abondant dans le plancton des deux étangs prospeclés.

- La présence de Macrocyclops fuscus et de M. albidus est très sporadique dans nos prélèvements planctoniques. Ces deux espèces préfèreraient les zones littorales à Macrophytes.

- Eucyclops lilljeborgi est assez fréquent dans Bogue en été.

- Paracyclops fimbriatus, considéré plutôt comme benthique, est rare dans nos prises de plancton.

1. Nous nous référons aux ouvrages de détermination de Dussart (1967 et 1969).

Nous remercions M. B. Dussart qui a bien voulu confirmer nos déterminations de Calanoida et Cyclopoida. 
TABI.EAU 3

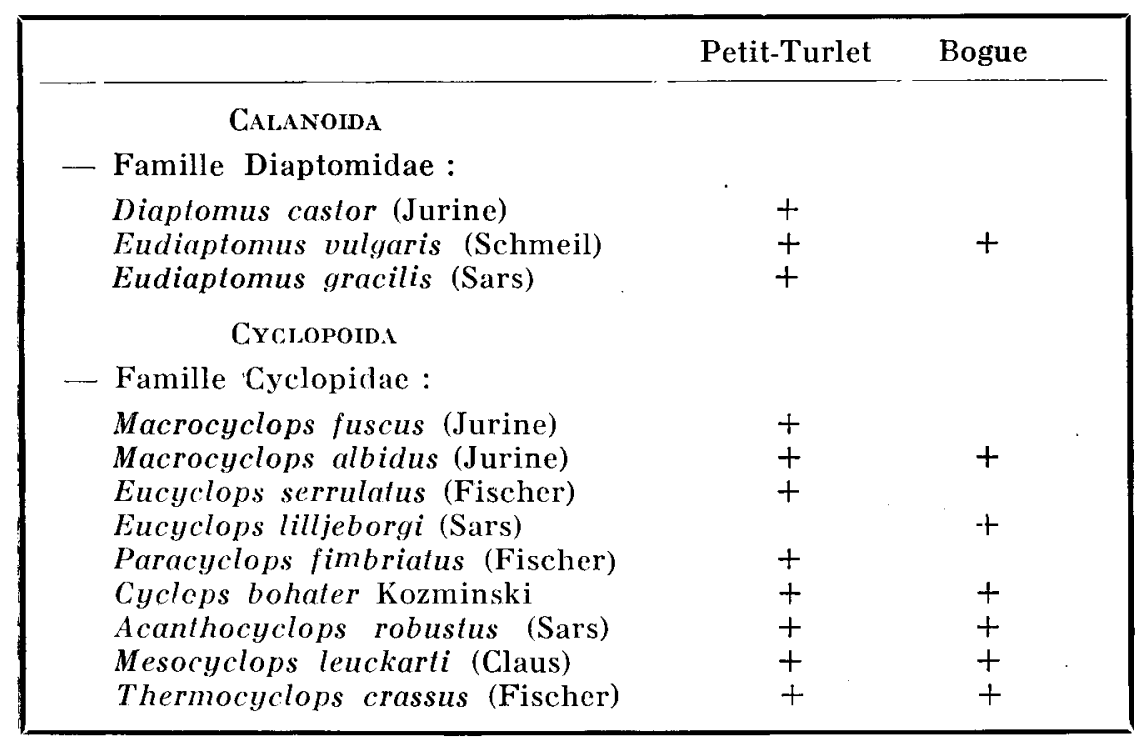

-- Cyclops bohater et Acanthocyclops robustus sont relativement communs dans les eaux de Petit-Turlet sauf en été.

- Par contre, Thermocyclops crassus, très rare dans Bogue, est hien représenté en été dans les eaux de Petit-Turlet.

- Les Harpacícoida sont peu communs; quelques individus capturés dans le benthon de ces deux étangs sont en cours de détermination.

\section{Les Nématodes.}

La détermination des espèces du genre Tobrilus tient compte de la mise au point récente de ANDrassy [1964]. Tobrilus consimilis (W. Schneider) est considéré comme synonyme de $T$. paludicola (Micoletzky).

La détermination d'une partie de la Nématofaune (Actinolaimidae, Alaimidae) encore à l'étude n'a porté que sur les genres; de nouvelles récoltes permettront une diagnose plus précise. Les Mermithidac présents à Petit Turlet et à Bogue n'ont pas été déterminés. 
Tableau 4

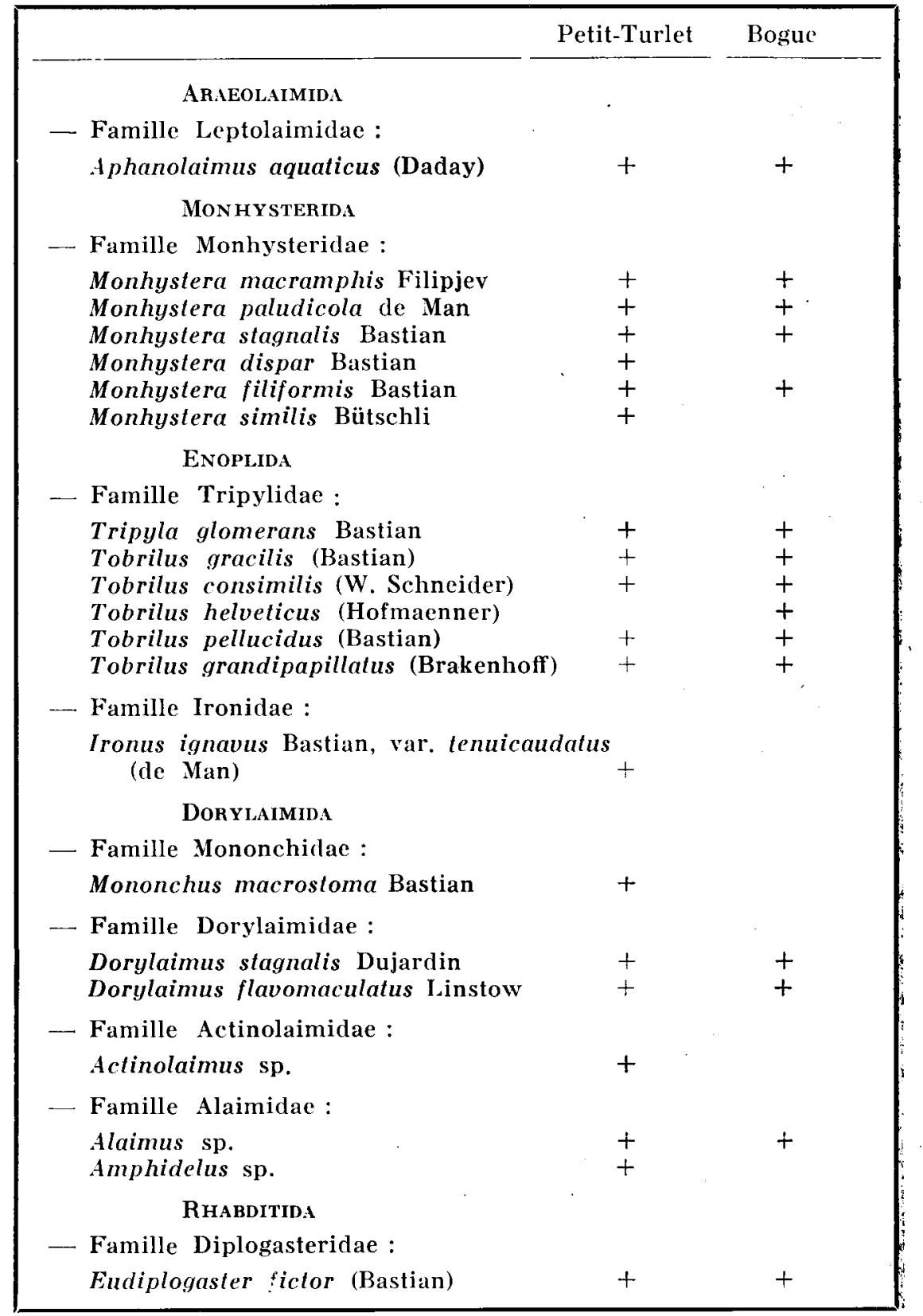


Les Oligochètes.

TABLEAU 5

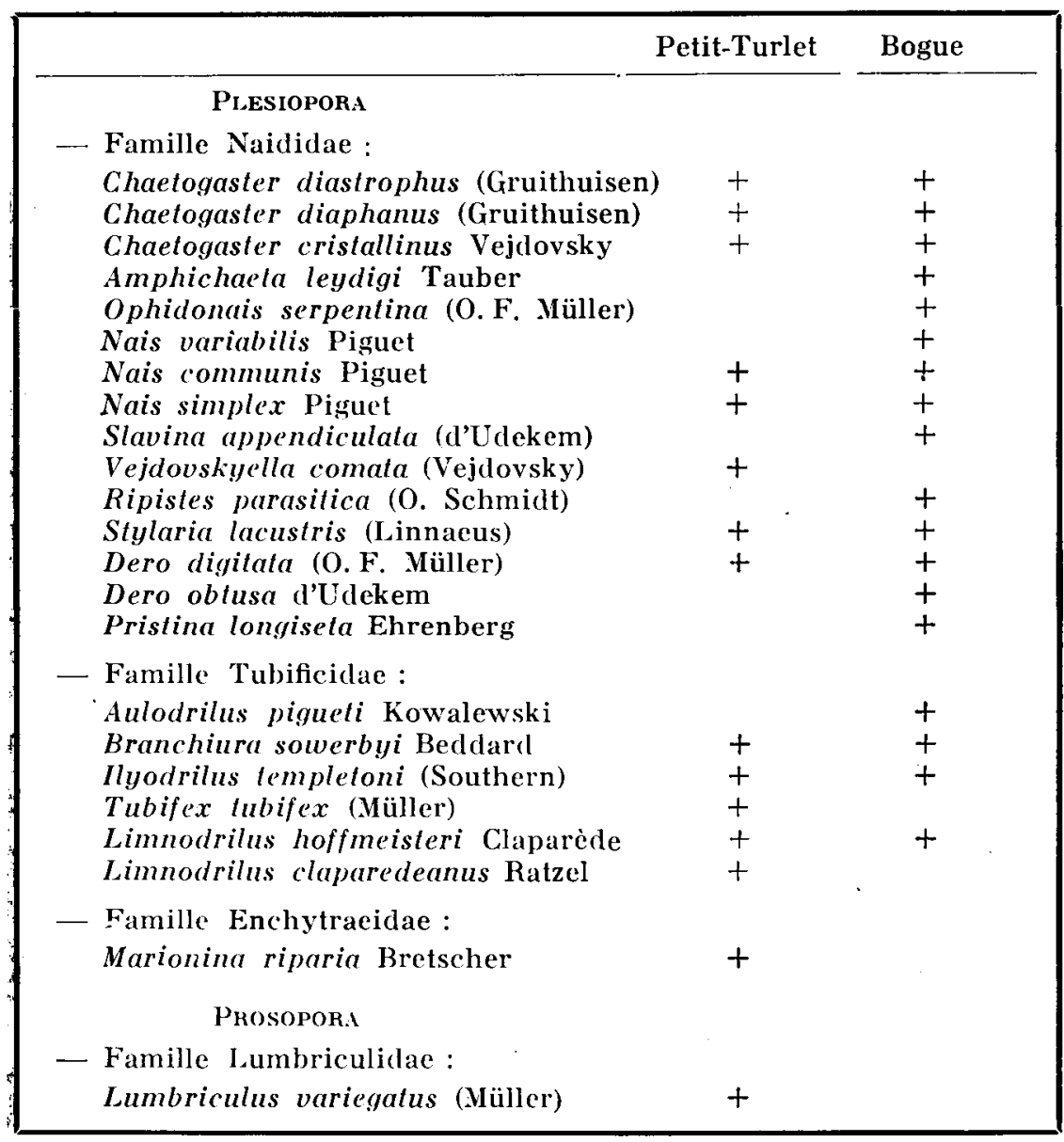

Plusieurs des espèces inventoriées hébergent divers parasites.

Limnodrilus hoffmeisteri est parasité par le Cestode Archigetes sieboldi Leuckart. Ilyodrilus templetoni et Dero obtusa hébergent (Ies Ciliés dans leur tube digestif. Une espèce de Rotifère vit également en endoparasite dans le tube digestif de Dero obtusa récoltée à Bogue.

- Remarques écologiques sur le benthon.

Ja comparaison de la faune benthique de Petit-Turlet et de Bogue appelle les remarques suivantes : 
a) Le bilan de l'oxygène dans l'étang de Bogue envahi par la végétation fait apparaître un déficit en $0^{2}$ principalement dans les couches d'eau les plus profondes et au niveau du sédiment. Ce déficit lié à l'absence prolongée d'assec et aux fermentations dues aux nombreux détritus d'origine végétale accumulés sur le fond explique l'abondance des larves de Chaoborus et l'association typique Chaoborus-Chirozomus plumosus. La fréquence du Nématode Tobrilus gracilis, espèce saprophile considérée comme un organisme indicateur de sédiments fortement réducteurs [ScHILMER, LöFFLER, Dollfuss, 1969], alors que cette espèce est beaucoup plus rare à Petit-Turlet, relèverait des mêmes causes. A l'appui de cette thèse on peut également invoquer la répartition de l'espèce Ironus ignavus, var. tenuicaudatus. Ce Nématode, considéré comme indicateur d'eaux $\beta$-mésosaprobes [Hirschmans, 1952], est abondant à Petit-Turlet mais paraît absent à Bogue.

b) La prédominance dans l'étang Bogue de la famille des Naididae dans le groupe des Oligochètes, aussi bien en ce qui concerne la densité des populations que le nombre des espèces, est liée à la richesse de cet étang en Macrophytes fixées ou flottantes. Cette famille comprend une majorité d'espèces rattachées au périphyton et dont certaines manifestent une prédilection pour les tourbières. Parmi les espèces communes à Bogue qui répondent à cette définition on peut citer Ophidonais serpentina, Slavina appendiculata, Ripistes parasitica, Pristina longiseta. Il est significatif que ces espèces n'aient pas été observées à Petit-Turlet dont la végétation est nettement moins abondante. Ces espèces trouvent un support adéquat non seulement parmi la végétation aquatique au cours de la bonne saison mais encore parmi les restes de racines, de rhizomes et de débris végétaux divers qui jonchent le fond de l'étang et qui leur servent de refuge surtout durant l'hiver. Ces débris organiques grossiers, faiblement dégradés, accumulés sur le fond entraînent la formation d'un horizon superficiel de consistance très souple, de quelques centimètres d'épaisseur, dont la structure tourbeuse contraste fortement avec la compacité des limons argileux sous-jacents. La biodégradation des polysaccharides s'y trouve ralentie du fait de l'acidité du milieu (favorisée par le pouvoir tampon des complexes argilo-humiques du sédiment) et de ses conditions asphyxiques ${ }^{1}$.

Par comparaison avec les sédiments de Bogue, les sédiments de Petit-Turlet dont les couches superficielles plus fines et plus homogènes renferment également un pourcentage plus important

1. La plus faible densité en microflore totale des sédiments de Bogue par rapport à celle des étangs voisins, notamment de Petit-Turlet [BEAUPIED, 1969], mérite également d'être signalée ici, car elle relève d'une origine commune. 
de matières organiques hébergent une population d'Oligochètes dominée par les Tubificidae. Cette famille renferme une majorité d'espèces limicoles parmi lesquelles dominent Ilyodrilus templetoni, Branchiura sowerbyi, Limnodrilus hoffmeisteri, Limnodrilus claparedeanus. A ce titre, l'étang Petit-Turlet peut être considéré comme un étang à Tubificidae, par opposition à l'étang Bogue où cette famille est supplantée par celle des Naididae.

\section{3. - CONCLUSIONS. PERSPECTIVES DE RECHERCHES}

L'inventaire préliminaire de la faune planctonique et benthique de 2 étangs de la Dombes, l'étang Petit-Turlet et l'étang Bogue, révèle l'importance des Crutacés et des Helminthes dans la produclion de ces 2 étangs à Cyprinidés.

Les Cladocères et les Oligochètes constituent en densité et en biovolume aussi bien que par le nombre des espèces présentes (respectivement 20 et 23 espèces) deux groupes systématiques prédominants. Les Copépodes, relativement abondants, comprennent surtout des représentants de l'ordre des Cyclopoida.

Parmi les éléments importants de la macrofaune dont l'étude n'a pas été abordée dans ce travail, il faut citer les Hydracariens et les Insectes, Diptc̀res et Ephéméroptères notamment (Ceratopogonidae, Tanypodinae, Chironomus, Chaoborus, Coenidae.

Les Nématodes (avec plus de 20 espèces) constituent l'un des groupes prédominants de la microfaune benthique des 2 étangs prospectés (exception faite des Protozoaires). Les Rotifères, les Gastrotriches, les Rhabdocoeles, les Tardigrades et les Ostracodes constituent d'autres éléments assez communs de la microfaune du périphyton et du benthon. Les Rotifères s'observent également en grand nombre dans le plancton de Bogue à certaines périodes.

L'apparente homogénéité du peuplement pourrait aisément s'expliquer par la proximité des 2 étangs et le mélange des populations favorisé par la richesse de l'avifaune locale dans le contexte particulier que constitue la Réserve Ornithologique de la Dombes [LEBRETon, 1965]. Des différences significatives affectent cependant le mode de répartition qualitative el quantitative des espèces et des groupes systématiques étudiés.

En raison de son caractère dystrophe (faible oxygénation, abondance de matières humiques), lié à l'absence d'assec, à la tendance climacique au comblement de la nappe d'eau par la végétation et à l'acidification corrélative du milieu, l'étang de Bogue, moins productif, abrite une bioçenose plus « spécialisée » que celle de Petit-Turlet. Ia faune de fond comporte notamment une association 
de formes saprophiles (ex. Chaoborus, Chironomus, Tobrilus gracilis) et d'espèces acidophiles propres aux tourbières (plusieurs espèces de Naididae).

L'étude faunistique des biocoenoses planctoniques et benthiques des étangs dombistes s'inscrit dans un programme de recherches d'écologie quantitative actuellement en cours. Ces recherches répondent plus précisément aux objectifs suivants ${ }^{1}$ :

a) Etude du cycle annuel des populations planctoniques et benthiques de 3 étangs de la Réserve Biologique de Dombes : l'étang Grand-Turlet, l'étang Petit-Turlet et l'étang Bogue. La mise en assec du Grand-Turlet au cours de l'année 1969 nous a permis d'entreprendre une série d'observations et d'expériences destinées à tester les capacités de survie et à évaluer le taux et la vitesse de repeuplement de cet étang en plancton et en benthon.

Lanalyse pédologique et microbiologique des sédiments de ces 3 étangs, amorcée par BEAUPIED [1969], est poursuivie, parallèlement à l'analyse physico-chimique de chaque nappe d'eau.

b) Etude du régime alimentaire et du taux de croissance de la Carpe qui représente l'élément principal de la production tertiaire des étangs piscicoles de la Dombes. Cette étude, entreprise dans l'étang Grand-Turlet, est destinée à évaluer, grâce à l'examen des contenus stomacaux, la qualité et la quantité de nourriture ingérée en fonction de l'âge et des saisons, ainsi que l'importance de la prédation sur l'évolution des populations planctoniques et benthiques.

\section{RÉSUMÉ}

L'inventaire systématique préliminaire des Crustacés et des Vers de l'étang Petit-Turlet (31 ha) et de l'étang Bogue (10 ha) montre l'importance quantitative des Cladocères et des Oligochètes respectivement dans le zooplancton et le zoobenthon. La différenciation des populations, surtout nette au niveau du benthon, est liée au régime propre d'exploitation de ces 2 étangs. I.e degré de comblement des nappes d'eau par les Macrophytes paludicoles et leurs résidus, favorisé le cas échéant par l'absence prolongée d'assec (étang Bogue), et, corrélativement, le degré d'acidité et les conditions d'oxygénation propres à chaque étang contribuent pour une large part à modeler directement ou indirectement la physionomie locale du peuplement.

1. Nous tenons à remercier M. P. FaURe, Directeur de la Régie départementale du Parc-Réserve de la Dombes, pour son accueil et les facilités qu'il a bien voulu nous accorder dans la prospection des étangs de la Réserve. 
PRELIMINARY STUDY OF THE ZOOPLANCTON AND ZOOBENTHOS FROM TWO FISH.PONDS IN THE DOMBES (AIN)

A preliminary study of Crustacea and Vermes from the ponds Petit-Turlet (31 ha) and Bogue (10 ha) showed the quantitative importance of Clarlocera in zooplancton and of Oligochaeta in zoobenthos. The differentiation of populations, clear at the benthos level, is connected with the type of management for the exploitation of these two ponds.

The local physiognomy of the fauna was correlated with the acidity and oxygenation in each pond, and was affected by the degree to which the water body was filled up by paludose macrophytes and their detritus, assisted by the prolonged absence of drought (Bogue pond).

\section{VORSTUDIEN DER PLANKTISCHEN UND BENTHISCHEN FAUNA ZWEIER FISCHTEICHE DER DOMBES (AIN)}

Eine vorläufige systematische Aufnahme der Krebstiere und Würmer des Petit-Turlet (31 ha) und des Bogue-Teiches $(10 \mathrm{ha})$ zeigt die quantitative Bedeutung der Cladoceren und der Oligochacten für das Zooplankton bzw. für das Zoobenthos.

Die Ausbildung verschiedener Populationen vor allem im Benthos ist mit der Art der Nutzung der Milieuofferte in diesen beiden Teichen verbunden. Das Ausmaß der Füllung der Wasserkörper durch Sumpfpflanzen und ihre Überreste im Falle des Bogue-Teiches, begünstigt durch das Fehlen einer längeren Periode der Trockenlegung, ferner ler Säuregrad und die jedem Teich eigenen Durchlüftungsbedingungen tragen weitgehend direkt oder indirekt zur Ausbildung der charakteristischen lokalen Besiedlung bei.

\section{TRAVAUX CITES}

Andrassy (I.). 1959. - Taxonomische Uebersicht der Dorylaimen (Nematoda), I. Acta Zool. Acad. Sc. Hungar., 5 (3-4) : 191-240.

ANdrassy (I.). 1964. - Ein Versuchsschlüssel zur Bestimmung der Tobrilus-Arten (Nematoda). Ann. Univ. Sc. Budapestin., Sect.Biol., $7: 3-18$.

BE.lupied (H.). 1969. - Contribution à l'étude microbiologique des sols d'étangs de la Dombes. Actes Réserve Biol. Dombes, 1967-1968: 27-83.

Brinkhurst (R.O.). 1963. - Taxonomic studies on the Tubificidae. Intern. Rev. Hydrobiol. Syst. Beith., 2 : 1-89.

Dussart (B.). 1967. -- Les Copépodes des eaux continentales. Tome I : Calanoides et Harpacticoides. Boubée, Paris, 500 p.

Dussart (B.). 1969. - Les Copépodes des eaux continentales. Tome II : Cyclopoides et biologie. Boubée, Paris, 292 p. 
Giuthier (M.). 1931. - Etude sur la composition planctonique de quelques étangs piscicoles des Dombes. Trav. Labo. Piscicult. Univ. Grenoble, 23 : $35-50$.

Gooney (T.). 1963. - Soil and freshwater Nematodes, (A monograph). Methuen, London, 544 p.

Gousden (C. E.). 1968. - The systematics and evolution of the Moinidae. Trans. Amer. Philos. Soc., 58 (6) : 101 p.

Hirschmany (H.). 1952. - Die Nematoden der Wassergrenze mittelfränkischer Gewässer. Zool. Jb. (Syst.), 81 : 313-436.

Joyeux (C. H.), BAER (J. G.). 1936. - Cestodes. In : Faune de France, Lechevalier, Paris, 30 : 613 p.

I.ebreton (P.). 1965. - La Réserve Biologique de Dombes (Ain). Nos Oiseaux, $28(299-300): 33-50$.

Levrat (R.). 1964-1965. - La pisciculture en Dombes. Bull. Fr. Piscicult., 37 (215) : 41-61; 37 (216) : 77-100; 37 (217) : 131-147; 38 (218) : 11-36.

Mas (P.). 1959. - Exploitation des étangs de la Dombes. Dipl. Ecole Nat. Agric. Montpellier, 140 p.

Meý (A. H.). 1960. - Freilebende Nematoden - In : Tierwelt Mitteleuropas, Quelle et Meyer, Leipzig, 1, 5 a, 164 p.

Nielsen (C. O.) et Christensex (B.). 1959. - The Enchytraeidae Critical revision and taxonomy of european species (studies on Enchytraeidae, 7). Natura Jutlandica, 8-9 : 1-160.

Pelosse (J.). 1926. - Sur la faune des Entomostracés de la région des Dombes. Congr. A.F.A.S., 50 session, Lyon : 429-432.

REy (J.). 1966. - Sur la récolte dans le Sud-Ouest de la France de Moina dubia de Guerne et Richard (Cladocères, Daphniidae). Ann. Limnol., 2 (1) : 177-182

Schiemer (F.), Loffler (H.) et Doldffuss (H.). 1969. - The benthic communities of Neusiedlersee (Austria), Verh. Int. Ver. Limnol., $17: 201-208$.

ScourfiELi) (D. J.). 1946. — A short-spined Daphnia presumably belonging to the longispina group - D. ambigua sp. n.J. Quekett Micr. Cl., 11 (4) : 127-131.

Sperber (C.). 1948. - A taxonomic study of the Naididae. Zool. Bidr., Uppsala, 28 : 1-296.

Ude (H.). 1929. - Würmer oder Vermes. I : Oligochaeta. In : Die Tierwelt Deutschl., 15 : 1-132.

Vivier (P.) et Wurtz (A.). 1960. - Les étangs de la Dombes. Rapp. Coll. Ass. Fr. Limnol., Fac. Sci. Lyon, inédit.

Wurtz (A.). 1956. - Ertragssteigerung in Teichen mit saurem Boden durch Haferkultur, Deutsche Fisch. Zeit., octobre 1956 : 306-313.

Wurtz (A.). 1960. - Le traitement du fond des étangs et ses effets sur la productivité. Stud. Rev. gen. Fish. Counc. Medit., 11 : $\mathrm{i}-\mathrm{ix}+\mathbf{1 - 1 9}$.

Wurtz (A.). 1962. - Mesures physico-chimiques et chimiques dans la vase et dans l'eau des étangs. Ann. Stat. Centr. Hydrobiol. Appl., 9 : 247-267.

(Section de Biologie Animale et Zoologie, Faculté des Sciences, Lyon.) 